\title{
Erratum to: Optimal solution for a cancer radiotherapy problem
}

\author{
A. Bertuzzi - C. Bruni · F. Papa - C. Sinisgalli
}

Published online: 27 December 2012

(C) Springer-Verlag Berlin Heidelberg 2012

\section{Erratum to: J Math Biol DOI 10.1007/s00285-012-0512-2}

Optimal schemes of five weekly radiation doses for a fractionated radiotherapy treatment have been studied in the original article as functions of tumour and healthy tissue parameters. The extremals and the optimal solutions derived there, however, did not account for "singular" situations in which some of the expressions (3.10), providing the elements of the extremal structures, are not defined.

For a given set of model parameters, singular situations occur when suitable KuhnTucker multipliers can be found so that the quantities $\delta, \sigma$ and $\tau$ defined in (3.1) satisfy the relations in one of the following cases:

The online version of the original article can be found under doi:10.1007/s00285-012-0512-2.

A. Bertuzzi · C. Sinisgalli $(\varangle)$

Istituto di Analisi dei Sistemi ed Informatica “A. Ruberti”,

CNR, Viale Manzoni 30, 00185 Roma, Italy

e-mail: carmela.sinisgalli@iasi.cnr.it

A. Bertuzzi

e-mail: sandro.bertuzzi@iasi.cnr.it

C. Bruni · F. Papa

Dipartimento di Informatica e Sistemistica "A. Ruberti",

Sapienza Università di Roma, Via Ariosto 25, 00185 Rome, Italy

e-mail: brunic@dis.uniroma1.it

F. Papa

e-mail: papa@dis.uniroma1.it 
(i) $\sigma=0, \quad \delta \geq 0$,

(ii) $\sigma \neq 0, \quad \tau \neq 0, \sigma= \pm \tau$,

(iii) $\sigma \neq 0, \quad \tau \neq 0, \sigma=\omega^{ \pm} \tau, \quad$ with $\omega^{ \pm}=\frac{1 \pm \sqrt{5}}{2}$.

Note that these cases are not mutually exclusive because each of them can occur, provided suitable multipliers $\eta_{e}, \eta_{l}$ exist.

If one of relations (i)-(iii) is verified, the system of five nonlinear equations used in the proof of Theorem 1 to get expressions (3.10) becomes indeterminate, so that the procedure outlined there does no longer provide the unknown doses $d_{i}, i=1,2, \ldots, 5$ as functions of $\delta, \sigma$ and $\tau$ only. Therefore, the set of possible extremal structures of Problem 1, detailed in Table 1 of Theorem 1, must include the additional singular structures listed below.

\section{Case (i)}

If $\delta=0$, the singular solutions are the points $d$ of the set

$$
\mathcal{S}=\left\{d \mid g_{e}(d)=g_{l}(d)=0, \sum_{i=2}^{5} d_{i-1} d_{i}=0, d_{i} \geq 0, i=1, \ldots, 5\right\} .
$$

If $\delta>0$, we have the singular structure

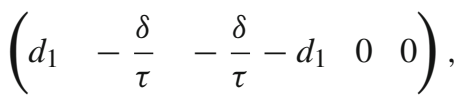

with $d_{1} \in[0,-\delta / \tau], \tau<0$.

\section{Case (ii)}

If $\sigma=\tau$, the representative singular structures to be added are

$$
\begin{aligned}
& \left(\begin{array}{lllll}
d_{1} & -\frac{\delta}{\tau}-d_{1} & 0 & 0 & 0
\end{array}\right), \\
& \left(\begin{array}{lllll}
-\frac{\delta}{\tau} & 0 & d_{3} & -\frac{\delta}{\tau}-d_{3} & 0
\end{array}\right), \\
& \left(\begin{array}{lllll}
d_{1} & -\frac{\delta}{\tau}-d_{1} & 0 & d_{4} & -\frac{\delta}{\tau}-d_{4}
\end{array}\right),
\end{aligned}
$$

with $d_{1}, d_{3}, d_{4} \in[0,-\delta / \tau], \delta \neq 0$ and $\delta, \tau$ of opposite sign. If $\sigma=-\tau$, we have two singular structures. The first one is

$$
\left(\begin{array}{lllll}
d_{1} & d_{1} & 0 & d_{4} & d_{4}
\end{array}\right),
$$

with $d_{1}, d_{4} \geq 0, \delta=0$, and the other

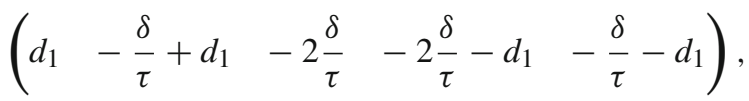

with $d_{1} \in[0,-\delta / \tau], \delta \neq 0$ and $\delta, \tau$ of opposite sign. 


\section{Case (iii)}

If $\sigma=\omega^{+} \tau$, we have

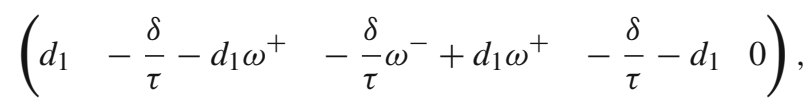

with $d_{1} \in\left[\left(\omega^{-}\right)^{2} \delta / \tau, \omega^{-} \delta / \tau\right], \delta \neq 0$ and $\delta, \tau$ of opposite sign.

If $\sigma=\omega^{-} \tau$, we have

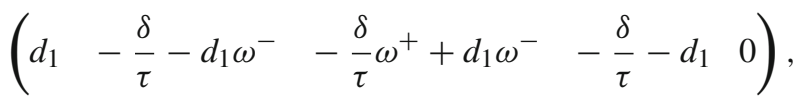

with $d_{1} \in[0,-\delta / \tau], \delta \neq 0$ and $\delta, \tau$ of opposite sign.

For any given set of radiobiological parameters of the tumour $(\rho, \gamma)$ and of the early $\left(\rho_{e}, \gamma_{e}, k_{e}\right)$ and late $\left(\rho_{l}, \gamma_{l}, k_{l}\right)$ responding tissues, the optimal solution must then be searched, besides the structures given in Theorem 1, among the singular structures listed above. The singular cases can actually provide extremals or even the optimum. As an example, let us consider tumours having $\rho$ in an "intermediate" range, i.e., $\rho \in\left[\rho_{l}, \rho_{e}\right]$, and $\gamma$ such that

$$
\mathrm{e}^{-\gamma} \leq \frac{\rho_{e}-\rho}{\rho_{e}-\rho_{l}} \mathrm{e}^{-\gamma_{l}}+\frac{\rho-\rho_{l}}{\rho_{e}-\rho_{l}} \mathrm{e}^{-\gamma_{e}}
$$

Any admissible solution $d$ for such tumours can be shown to have a cost function $J$ that verifies

$$
J(d) \geq-\frac{\rho_{e}-\rho}{\rho_{e}-\rho_{l}} k_{l}-\frac{\rho-\rho_{l}}{\rho_{e}-\rho_{l}} k_{e}
$$

It can be shown that all the points $d$ of the set $\mathcal{S}$ in Eq. (1) (assumed nonempty), with multipliers

$$
\eta_{e}=\frac{\rho-\rho_{l}}{\rho_{e}-\rho_{l}}, \quad \eta_{l}=\frac{\rho_{e}-\rho}{\rho_{e}-\rho_{l}},
$$

are optimal since they satisfy the necessary conditions (3.2)-(3.9) with $\sigma=\delta=0, \tau \geq 0$ and have the minimum value of $J$ :

$$
J(d)=-\frac{\rho_{e}-\rho}{\rho_{e}-\rho_{l}} k_{l}-\frac{\rho-\rho_{l}}{\rho_{e}-\rho_{l}} k_{e}, \quad d \in \mathcal{S} .
$$

Furthermore, it can be proved that $\mathcal{S} \neq \varnothing$ if and only if $v \in[1,3]$, where $v$ is defined in (4.12).

In the absence of the incomplete repair term, i.e., $\tau=0$ (see Section 4), only the singular case (i) with $\delta=0$ can occur. The related singular solutions can exist only for $\rho \in\left[\rho_{l}, \rho_{e}\right]$. The singular solutions set $\tilde{\mathcal{S}}$ is defined by

$$
\tilde{\mathcal{S}}=\left\{d \mid \tilde{g}_{e}(d)=\tilde{g}_{l}(d)=0, d_{i} \geq 0, i=1, \ldots, 5\right\},
$$


with $\tilde{g}_{e}$ and $\tilde{g}_{l}$ as in Eq. (4.2), and it is nonempty if and only if $k_{e}-k_{l}>0, v \in[1,5]$. The cost function $\tilde{J}$ in (4.1), evaluated for $d \in \tilde{\mathcal{S}}$, has the same expression of the r.h.s. of (3) and since $\tilde{J}$ again satisfies inequality (2), we conclude that the singular solutions $d \in \tilde{\mathcal{S}}$ are optimal for $\rho \in\left[\rho_{l}, \rho_{e}\right], k_{e}-k_{l}>0$ and $v \in[1,5]$. Therefore, for such values of $\rho, k_{e}, k_{l}$ and $v$, Table 4 must be corrected by changing the entry $d_{e}^{([v]+1)}$ to $\tilde{\mathcal{S}}$ and Table 5 by adding the points of $\tilde{\mathcal{S}}$ to the optimal solutions.

For the problem considered in Section 5 (prevalent late constraint), we analytically verified by direct comparison with the cost function of the optimal solutions listed in Theorem 6 that no singular solution can be optimal, so that all the results given there still hold.

The numerical results reported in Tables 6-9 of Section 6 are not modified. Those Tables, in fact, refer to the problem in the absence of the incomplete repair term $(\tau=0)$ with $v=5$, where the set $\tilde{\mathcal{S}} \equiv d^{(5)}$. Moreover, the results in Tables $10-13$ for the problem with prevalent late constraint still hold. Finally, all the results of Tables 14 and 15 are also valid since for the chosen parameter values the singular cases do not provide any additional extremal. 